\title{
Relationship Between Fatigue and Inflammation, Disease Duration, and Chronic Pain in Psoriatic Arthritis: An Observational DANBIO Registry Study
}

\author{
Marie Skougaard (D), Tanja Schjødt Jørgensen (1D, Signe Rifbjerg-Madsen, Laura C. Coates (D, \\ Alexander Egeberg, Kirstine Amris (1), Lene Dreyer, Pil Højgaard (D), Jørgen Guldberg-Møller (D), \\ Joseph F. Merola, Peder Frederiksen, Henrik Gudbergsen (1), and Lars Erik Kristensen (1)
}

\begin{abstract}
Objective. Fatigue is one of the most significant symptoms, and an outcome of great importance, in patients with psoriatic arthritis (PsA), but associations between underlying components of fatigue experienced by patients in relation to the disease have been sparsely investigated. The objectives were to describe the degree of fatigue in patients with PsA, and to examine important components associated with fatigue.
\end{abstract}

Methods. We performed a cross-sectional survey including patients registered in the Danish nationwide registry DANBIO from December 2013 to June 2014. Principal component analysis (PCA) was used to identify factors associated with fatigue.

Results. A total of 1062 patients with PsA were included in the study. A PCA reduced co-variables into 3 components explaining $63 \%$ of fatigue in patients. The first component, contributing to $31 \%$ of fatigue, was composed of inflammatory factors including swollen and tender joints, physician's global assessment, elevated C-reactive protein (CRP), and high Pain Detect Questionnaire (PDQ) score. The second component, contributing to $17 \%$ of fatigue, consisted of increasing age and long disease duration. The third component, contributing to $15 \%$ of fatigue, consisted of high PDQ score, tender joint count, increasing age, and concomitant low CRP, suggestive of a chronic pain component consisting of central pain sensitization or structural joint damage.

Conclusion. Fatigue in patients with PsA may be driven by clinical inflammatory factors, disease duration, and chronic pain in the absence of inflammation. (First Release December 15 2019; J Rheumatol 2020;47:548-52; doi:10.3899/jrheum.181412)

Key Indexing Terms:

PSORIATIC ARTHRITIS

FATIGUE

PRINCIPAL COMPONENT ANALYSIS

From The Parker Institute, and the Department of Rheumatology, Copenhagen University Hospital, Bispebjerg and Frederiksberg, Copenhagen, Denmark; Nuffield Department of Orthopaedics, Rheumatology and Musculoskeletal Sciences, University of Oxford, Oxford, UK; Department of Dermatology and Allergy, Copenhagen University Hospital Gentofte and Herlev, Gentofte; departments of Clinical Medicine and Rheumatology, Aalborg University and Aalborg University Hospital, Aalborg; The DANBIO Registry, Center for Rheumatology and Spine Diseases, Centre for Head and Orthopaedics, Rigshospitalet, Glostrup; the Center for Rheumatology and Spine Diseases, Copenhagen University Hospital Gentofte and Herlev, Gentofte, Denmark; Department of Dermatology and Department of Medicine, Division of Rheumatology, Brigham and Women's Hospital, Harvard Medical School, Boston, Massachusetts, USA.

This work was supported by a core grant from the Oak Foundation [OCAY-13-309]. The research was supported by the UK National Institute for Health Research (NIHR) Oxford Biomedical Research Centre (BRC). L.C. Coates is funded by an NIHR Clinician Scientist award. The views expressed are those of the author(s) and not necessarily those of the UK National Health Service, the NIHR, or the Department of Health. T.S. Jørgensen is on the speaker's bureau for AbbVie, Roche, UCB, Novartis, and Biogen. L.C. Coates has received grant/research support from AbbVie, BMS, Celgene, Jannsen Pharmaceuticals, Lilly, MSD, Novartis, Pfizer, Sun Pharma, and UCB. A. Egeberg has received grant/research support from Pfizer and Eli Lilly, and is on the speaker's bureau for Almirall, Samsung Bioepis Co., Pfizer, Eli Lilly, Novartis, Galderma, and Janssen Pharmaceuticals. L. Dreyer is on the speaker's bureau for MSD, UCB, and Janssen Pharmaceuticals. P. Højgaard is on the speaker's bureau of Celgene, UCB, and J. Guldberg-Mфller, and is a paid instructor for AbbVie. J. Merola has received grant/research support from Biogen IDEC, Amgen, Pfizer, and Boehringer Ingelheim, and is a consultant for Biogen IDEC, Eli Lilly, Novartis, Momenta, UCB, Kiniksa, AbbVie, Amgen, Pfizer, Janssen Pharmaceuticals, Momenta, and Mallinckrodt, and is on the speaker's bureau for AbbVie. H. Gudbergsen is on the speaker's bureau for MSD and Pfizer. L.E. Kristensen is on the speaker's bureau of Pfizer, AbbVie, Amgen, UCB, Celgene, BMS, MSD, Novartis, Eli Lilly, and Janssen Pharmaceuticals.

M. Skougaard, MD, The Parker Institute, Copenhagen University Hospital, Bispebjerg and Frederiksberg; T.S. Jorgensen, MSc, PhD, The Parker Institute, Copenhagen University Hospital, Bispebjerg and Frederiksberg; S. Rifbjerg-Madsen, $M D, P h D$, The Parker Institute, and the Department of Rheumatology, Copenhagen University Hospital, Bispebjerg and Frederiksberg; L.C. Coates, MBChB, MRCP, PhD, Nuffield Department of Orthopaedics, Rheumatology and Musculoskeletal Sciences, University of Oxford; A. Egeberg, MD, PhD, Department of Dermatology and Allergy, Copenhagen University Hospital Gentofte and Herlev; K. Amris, MD, DMsc, The Parker Institute, Copenhagen University Hospital, Bispebjerg and Frederiksberg; L. Dreyer, MD, Professor, The Parker Institute, Copenhagen University Hospital, Bispebjerg and Frederiksberg, and the departments of Clinical Medicine and Rheumatology, Aalborg University and Aalborg University Hospital, and the DANBIO Registry, Center for Rheumatology and Spine Diseases, Centre for Head and Orthopaedics, Rigshospitalet; P. Højgaard, MD, PhD, The Parker Institute, Copenhagen University Hospital, Bispebjerg and Frederiksberg, and Center for Rheumatology and Spine Diseases, Copenhagen University Hospital Gentofte and Herlev; 
Psoriatic arthritis (PsA) is a chronic inflammatory musculoskeletal disease with a prevalence of $0.2 \%$ in Denmark ${ }^{1}$. The disease confers a considerable socioeconomic disease burden with decreased work productivity and increased healthcare use ${ }^{2,3}$. Moreover, patients with PsA are characterized by a decreased quality of life compared to other patient groups and often fatigue is reported to be the factor limiting participation in daily activities ${ }^{4,5}$.

Fatigue defined as sustained physical tiredness, mental exhaustion, and a lack of energy, is a well-known symptom of many chronic diseases ${ }^{6,7}$ and is often a crucial aspect in the management of such diseases ${ }^{8}$. It is a common symptom in PsA and is deemed by patients to be one of the most significant symptoms ${ }^{9,10}$. It is rated by patients as the worst symptom after pain and skin problems ${ }^{7,9,11}$.

Though fatigue is considered an important outcome measure for patients with PsA, this outcome is not yet fully embedded in clinical practice or in the scientific thinking within this disease area, where reporting of fatigue as a patient-reported outcome is rare and studies on fatigue are limited ${ }^{7,12}$.

However, the focus on fatigue is increasing and fatigue is now considered a core outcome according to the updated PsA core domain set from $2016^{13}$. Studies have described the association between fatigue in patients with PsA and pain, female sex, physical disability, medication status, psychological distress, longstanding sick leave, and loss of ability to work ${ }^{8,11}$. Further, biological agents have been shown to improve fatigue, suggesting a link between fatigue and inflammatory signaling $14,15,16,17,18,19$. And so the inflammatory pathway is believed to be associated with several clinical manifestations of PsA. As for pain in PsA, it is traditionally considered to be of inflammatory origin, but despite better control of inflammation, some patients still report pain as a significant concern. This suggests that PsA may prompt central sensitization and thus be linked to other central mechanisms such as fatigue, and indicates why it is relevant to study the quality of pain [i.e., by using the Pain Detect Questionnaire (PDQ) rather than just measuring quantity in terms of visual analog scale (VAS) pain].

The objective of our study was to describe the degree of fatigue in patients with PsA in a nationwide study, and to examine important components associated with fatigue.

J. Guldberg-Møller, MD, The Parker Institute, Copenhagen University Hospital, Bispebjerg and Frederiksberg; J.F. Merola, MD, MMSc, Department of Dermatology and Department of Medicine, Division of Rheumatology, Brigham and Women's Hospital, Harvard Medical School; P. Frederiksen, MSc, The Parker Institute, Copenhagen University Hospital, Bispebjerg and Frederiksberg; H. Gudbergsen, $M D, P h D$, The Parker Institute, Copenhagen University Hospital, Bispebjerg and Frederiksberg; L.E. Kristensen, $M D, P h D$, The Parker Institute, Copenhagen University Hospital, Bispebjerg and Frederiksberg.

Address correspondence to Dr. L.E. Kristensen, The Parker Institute, Copenhagen University Hospital Bispebjerg and Frederiksberg, Nordre Fasanvej 57, Road 8, Entrance 19, 2000 Frederiksberg, Denmark. E-mail:lars.erik.kristensen@regionh.dk

Accepted for publication June 27, 2019.

\section{MATERIALS AND METHODS}

Study design and setting. The study was designed as a cross-sectional survey including patients registered in the Danish nationwide registry, $\mathrm{DANBIO}^{20}$. Recording of data in DANBIO was mandatory for patients in treatment with biological disease-modifying antirheumatic drugs (bDMARD), but DANBIO also contains treatment information on patients treated with conventional synthetic DMARD (csDMARD). PDQ was implemented on the DANBIO touch screens in Danish outpatient clinics at 22 of 24 departments of rheumatology for a period of 6 months (December 1, 2013, to June $1,2014)$. The study was conducted in accordance with the STROBE statement (Supplementary Figure 1, available with the online version of this article) and according to a prespecified protocol available and published as open access at the official Website of the Parker Institute, a research unit that is part of Copenhagen University Hospital at Bispebjerg and Frederiksberg (www.parkerinst.dk). All patients registered as having PsA were invited to participate in the survey. Patients with a complete response to PDQ and a PDQ score above 0 were included in the analyses. Patient consent was obtained on the touch screen prior to the redirection to the PDQ. In accordance with Danish legislation, surveys do not require approval by ethics committees. Registrations and publications of data from clinical registries that do not pertain to human biological samples do not require patient consent or approval by ethics committees.

Variables and outcome measures. The VAS is a single-item scale (0-100 $\mathrm{mm}$ ) to measure patient-reported pain, fatigue, and global health (VAS pain, VAS fatigue, VAS global health). The VAS scale has shown good reliability and performs as well as other questionnaires when assessing fatigue ${ }^{21}$. In this study the VAS was used to measure patient-reported fatigue during the last week, with "0" representing "no fatigue" and " 100 " representing "worst imaginable fatigue" 22 . We defined moderate-to-severe fatigue as fatigue scores $\geq 57$ (chosen because 57 was the median VAS fatigue score for the population). PDQ is a mechanism-based pain classification instrument based on patient self-reported somatosensory signs and symptoms, assigning patients to one of 3 categories depending on the character of the experienced pain: neuropathic (PDQ score $>18$ ), unclear (PDQ score 13-18), or nociceptive (PDQ score < 13). PDQ was originally developed to screen for a neuropathic pain component ${ }^{23}$ and based on pain phenotypic similarities to assess neuropathic pain features as a proxy of central sensitization ${ }^{23,24,25}$. Statistical analysis. Patient characteristics were given with median and interquartile ranges (IQR) for continuous variables. Spearman's $\rho$ correlation coefficients were calculated to assess any potential association between fatigue scores and clinical indices. Two-sided $p$ values $<0.05$ were regarded as statistically significant.

To examine components explaining fatigue, a principal component analysis (PCA) was conducted. Variables were a priori selected based on clinical relevance with a predefined maximum allowed collinearity of 0.4 . Variables included for further analysis consisted of age, disease duration, swollen/tender joint count (28 joints), pain detect score, C-reactive protein (CRP) level, and patient and doctor VAS global health scores $(0-100 \mathrm{~mm})$. Health Assessment Questionnaire (HAQ) scores were excluded from the PCA owing to collinearity. To assess the variability and association of components to fatigue in the entire population, multiple linear regression was conducted for VAS fatigue, with the 3 primary components identified in the PCA. A sensitivity analysis based on the PCA was constructed on VAS pain and stratification by sex, respectively, to explore any possible similarities or differences explaining fatigue when including PDQ score versus VAS pain and male versus female. IBM SPSS version 20 was used to carry out the analyses.

\section{RESULTS}

A total of 2388 patients were diagnosed with PsA in DANBIO, of which 2114 had a VAS fatigue score. Of these, 1062 chose to participate in the study and were included for analysis because they had a recorded PDQ score above 0 . The

Personal non-commercial use only. The Journal of Rheumatology Copyright $\subset$ 2020 . All rights reserved. 
median VAS fatigue score was $57 \mathrm{~mm}$ for the population, and scores of $57 \mathrm{~mm}$ or more were considered moderate to severe fatigue. Patients with moderate to severe fatigue were predominantly female, and with higher 28-joint Disease Activity Score (DAS28)-CRP as well as higher VAS pain, VAS global health, PDQ score, and HAQ scores, compared with subjects with none to mild fatigue scores. Moreover, these patients had higher scores in physician's global assessment (PGA), more tender and swollen joints, increased use of corticosteroids, and more often switched bDMARD (Table 1).

In the PCA (Supplementary Figure 2, available with the online version of this article), the clinical co-variables were reduced to 3 components explaining 63\% of fatigue (Figure 1 ). The first component, contributing to $31 \%$, was mainly constituted by inflammatory factors such as more swollen and tender joints, higher PGA, higher DAS28-CRP, and higher PDQ scores, whereas the second component mainly consisted of contributions from older age and longer disease duration, explaining $17 \%$ of fatigue. The third component, contributing to $15 \%$, consisted of higher PDQ scores, more tender joint counts, increasing age, and concomitant low CRP.

The multiple linear regression analysis on the overall population with VAS fatigue as the dependent variable and the 3 identified components as independent variables showed an overall significant association of increasing fatigue with a correlation coefficient of 0.39 ( $p$ value $<0.001$ ). For the first and third component the correlation coefficients were 0.73 and 0.35 , respectively, with statistically significant $\mathrm{p}$ value $<0.001$. For the second component, the regression coefficient was 0.06 , with a $p$ value of 0.45 . In the sensitivity analysis, the PCA reduced the clinical co-variables to 3 major components explaining $64 \%$ of experienced fatigue (Supplementary Figure 3, available with the online version of this article). The components identified that included VAS pain in the analysis were almost identical to the components identified that included PDQ score. Comparing PCA performed on male versus female also resulted in similar components explaining $68 \%$ and $61 \%$ of experienced fatigue, respectively, though with a difference from the primary PCA in the inflammatory component; $36 \%$ in males and $29 \%$ in females (Supplementary Figure 4).

\section{DISCUSSION}

The median fatigue score in this population-based PsA cohort including patients treated with csDMARD and bDMARD was $\geq 57 \mathrm{~mm}$ VAS, underscoring the great importance of fatigue as a patient-reported disease manifestation. Our findings from the PCA in the population with fatigue above

Table 1. Patient characteristics

\begin{tabular}{|c|c|c|c|c|c|}
\hline \multirow[t]{2}{*}{ Characteristics } & \multicolumn{2}{|c|}{$\begin{array}{c}\text { Fatigue: None to Mild, } \\
\text { VAS Score }<57, \mathrm{n}=520\end{array}$} & \multicolumn{2}{|c|}{$\begin{array}{l}\text { Fatigue: Moderate to Severe, } \\
\text { VAS Score } \geq 57, n=542\end{array}$} & \multirow[t]{2}{*}{$\mathrm{p}$} \\
\hline & & $\mathrm{n}$ & & $\mathrm{n}$ & \\
\hline Female, n (\%) & $253(48.7)$ & 520 & $358(66.1)$ & 542 & $<0.001$ \\
\hline Age, yrs & $53.0(44.0-62.0)$ & 520 & $52.0(42.8-60.0)$ & 542 & 0.070 \\
\hline Disease duration, yrs & $6.0(3.0-11.5)$ & 449 & $5.0(2.0-10.0)$ & 456 & 0.022 \\
\hline Previous use of DMARD, n (\%): & & 520 & & 542 & 0.046 \\
\hline None & $449(86.3)$ & & $443(81.7)$ & & \\
\hline 1 & $44(8.5)$ & & $50(9.2)$ & & \\
\hline 2 & $26(5.0)$ & & $49(9.0)$ & & \\
\hline $3+$ & $1(0.1)$ & & $0(0.0)$ & & \\
\hline Use of MTX, n (\%) & $316(60.8)$ & 520 & $313(57.7)$ & 542 & 0.319 \\
\hline Concomitant corticosteroid, n (\%) & $6(1.2)$ & 520 & $29(5.4)$ & 542 & $<0.001$ \\
\hline Biological treatment, status, n (\%) & & 520 & & 542 & $<0.001$ \\
\hline Never treated with biologicals & $272(52.3)$ & & $279(51.5)$ & & \\
\hline In current treatment & $224(43.1)$ & & $195(36.0)$ & & \\
\hline Previous use & $24(4.6)$ & & $68(12.5)$ & & \\
\hline SJC, $0-28 *$ & $0.47 \pm 1.3$ & 455 & $0.94 \pm 2.2$ & 459 & $<0.001$ \\
\hline TJC, $0-28 *$ & $1.73 \pm 3.6$ & 456 & $5.0 \pm 6.4$ & 469 & $<0.001$ \\
\hline $\mathrm{CRP}, \mathrm{mg} / \mathrm{l}$ & $3.0(1.0-6.0)$ & 421 & $4.0(2.0-7.0)$ & 464 & 0.008 \\
\hline Patient pain assessment, $0-100 \mathrm{~mm}$ VAS & $25.0(15.0-38.0)$ & 520 & $66.0(49.0-78.0)$ & 542 & $<0.001$ \\
\hline PtGA, 0-100 mm VAS & $27.0(15.0-43.0)$ & 520 & $75.5(61.0-86.0)$ & 542 & $<0.001$ \\
\hline PGA, 0-100 mm VAS & $7.0(3.0-15.0)$ & 432 & $14.0(7.0-14.0)$ & 438 & $<0.001$ \\
\hline PDQ score & $9.0(6.0-14.0)$ & 520 & $17.0(13.0-23.0)$ & 542 & $<0.001$ \\
\hline DAS28-CRP & $2.3(1.8-2.9)$ & 400 & $3.5(2.6-4.4)$ & 418 & $<0.001$ \\
\hline HAQ score, 0-3 & $0.4(0.1-0.8)$ & 507 & $1.1(0.8-1.6)$ & 530 & $<0.001$ \\
\hline
\end{tabular}

Unless otherwise stated, data were given as median with interquartile range. $*$ SJC and TJC given as mean \pm SD. VAS: visual analog scale; DMARD: disease-modifying antirheumatic drugs; MTX: methotrexate; PDQ: Pain Detect Questionnaire; CRP: C-reactive protein; DAS28-CRP: 28-joint count Disease Activity Score using CRP; HAQ: Health Assessment Questionnaire; SJC: swollen joint count; TJC: tender joint count; PtGA: patient's global assessment; PGA: physician's global assessment. 


\section{Component 1}

Clinical inflammatory

manifestations
Loading factors of included variables:

- Doctor's VAS: 0.82*

- Swollen joints: $0.77^{*}$

- Tender joints: 0.73*

- PDQ: 0.41*

- CRP: 0.41*

- Disease duration: -0.08

- Age: -0.05

\section{Component 2}

Chronicity

\section{Component 3} Chronic pain
Loading factors of included variables:

- Disease duration: 0.74*

- Age: 0.66*

- Swollen joints: 0.21

- CRP: 0.08

- Doctor's VAS: 0.07

- Tender joints: -0.01

- PDQ: $-0.38^{*}$

Loading factors of included variables:

- PDQ: 0.59*

- Age: $0.43^{*}$

- Tender joints: $0.35^{*}$

- Disease duration: 0.04

- Swollen joints: -0.08

- Doctor's VAS: -0.20

- CRP: $-0.61^{*}$

Figure 1. Principal component analysis indicating 3 components explaining fatigue. The 3 components explaining fatigue include (1) clinical inflammatory manifestations, (2) chronicity, and (3) chronic pain. * High-impact variables contributing to the component. Each variable is presented with the corresponding loading factor. VAS: visual analog scale; CRP: C-reactive protein; PDQ: Pain Detect Questionnaire.

the median suggested that fatigue was constituted by an inflammatory component, disease duration, and chronic pain in the absence of inflammation. Moreover, the multiple linear regression analysis showed that there was a significant and clinically relevant association with the 3 components and increasing fatigue in the entire population.

Conducting the PCA led to 3 components that affected and explained $63 \%$ of experienced moderate to severe fatigue in patients with PsA. The first component was driven by clinical inflammatory factors such as DAS28-CRP, PGA, and swollen and tender joints, revealing one of the underlying explanations of fatigue to be actual inflammatory disease activity - highlighting the importance of targeted treatment of PsA. The second component consisted of disease duration and age, leading our attention to the important aspect of a link between fatigue and disease chronicity. The third component was defined by an inverse relationship between low CRP and high pain indicators. High PDQ scores in the moderate to severe fatigue group suggested central pain sensitization, though the contribution from tender joints to the third component might be explained by a degree of structural damage as well ${ }^{26}$. When substituting PDQ scores with VAS pain, the same components were identified, underscoring the experienced pain as an important driver of fatigue independent of cause or origin for the pain. PDQ scores were in general higher in patients with moderate to severe fatigue, implying a higher degree of centrally derived pain in this group. Chronic pain conditions are common within rheumatic diseases and this further indicates the importance of differentiating patients to provide them with the best possible care.

Previous studies showed that bDMARD and targeted treatments improved symptoms of fatigue in patients with PsA compared to placebo-controlled groups ${ }^{14,17,19}$, indicating an inflammatory component in the type of fatigue also found in our present study. From the percentages experiencing no change in fatigue ${ }^{18,19}$, one could consider whether this to a degree is treatment-refractory because of other components influencing experienced fatigue.

In line with previous research ${ }^{11}$, our present study found that the moderate to severe fatigue group consisted of a statistically significant higher number of females, had higher pain scores, and higher HAQ scores. Additionally, the present study also found that concomitant use of corticosteroids and patients more often switching bDMARD were associated with having moderate to severe fatigue.

Limitations of our current study were (1) the incompleteness of baseline data; however, the proportion of missing data did not exceed $25 \%$ for any variable, and (2) the risk for

Personal non-commercial use only. The Journal of Rheumatology Copyright $\subset$ 2020 . All rights reserved. 
selection bias of the patients because recording of data in DANBIO was mandatory only for patients treated with bDMARD, which may lead to overrepresentation of patients with more severe disease who were taking highly effective therapies. Nonetheless, pain and fatigue remain of utmost importance to patients, and the current study offers new insights into the mechanisms leading to fatigue.

Our study showed a strong association between fatigue and clinically important features including inflammation, disease duration, and chronic pain, which are relevant to take into account when treating PsA. The 3 components explained in total $63 \%$ of the experienced fatigue in the moderate to severe fatigue population of patients with PsA.

\section{ACKNOWLEDGMENT}

We acknowledge our patient research partner for taking part in the process of preparing the study through discussions of fatigue in relation to PsA and for reading and commenting on the final article.

\section{ONLINE SUPPLEMENT}

Supplementary material accompanies the online version of this article.

\section{REFERENCES}

1. Egeberg A, Kristensen LE, Thyssen JP, Gislason GH, Gottlieb AB, Coates LC, et al. Incidence and prevalence of psoriatic arthritis in Denmark: a nationwide register linkage study. Ann Rheum Dis 2017;76:1591-7.

2. Cortesi P, Scalone L, D’Angiolella L, Belisari A, Fusco F, Olivieri I, et al. Systematic literature review on economic implications and pharmacoeconomic issues of psoriatic arthritis. Clin Exp Rheumatol 2012;30:126-31

3. Sokoll KB, Helliwell PS. Comparison of disability and quality of life in rheumatoid and psoriatic arthritis. J Rheumatol 2001;28:1842-6.

4. Rosen CF, Mussani F, Chandran V, Eder L, Thavaneswaran A, Gladman DD. Patients with psoriatic arthritis have worse quality of life than those with psoriasis alone. Rheumatology 2011;51:571-6.

5. Walsh JA, McFadden ML, Morgan MD, Sawitzke AD, Duffin KC, Krueger GG, et al. Work productivity loss and fatigue in psoriatic arthritis. J Rheumatol 2014;41:1670-4.

6. Chandran V, Bhella S, Schentag C, Gladman DD. Functional assessment of chronic illness therapy-fatigue scale is valid in patients with psoriatic arthritis. Ann Rheum Dis 2007;66:936-9.

7. Gossec L, de Wit M, Kiltz U, Braun J, Kalyoncu U, Scrivo R, et al. A patient-derived and patient-reported outcome measure for assessing psoriatic arthritis: elaboration and preliminary validation of the Psoriatic Arthritis Impact of Disease (PSAID) questionnaire, a 13-country EULAR initiative. Ann Rheum Dis 2014;73:1012-9.

8. Skoie IM, Ternowitz T, Jonsson G, Norheim K, Omdal R. Fatigue in psoriasis: a phenomenon to be explored. Br J Dermatol 2015;172:1196-203.

9. Orbai A-M, Mease PJ, De Wit M, Kalyoncu U, Campbell W, Tillett W, et al. Report of the GRAPPA-OMERACT Psoriatic Arthritis Working Group from the GRAPPA 2015 annual meeting. J Rheumatol 2016;43:965-9.

10. Cella D, Wilson H, Shalhoub H, Revicki DA, Cappelleri JC, Bushmakin AG. Content validity and psychometric evaluation of
Functional Assessment of Chronic Illness Therapy-Fatigue in patients with psoriatic arthritis. J Patient Rep Outcomes 2019;3:5.

11. Husted JA, Tom BD, Schentag CT, Farewell VT, Gladman DD Occurrence and correlates of fatigue in psoriatic arthritis. Ann Rheum Dis 2009;68:1553-8.

12. Palominos PE, Gaujoux-Viala C, Fautrel B, Dougados M, Gossec L. Clinical outcomes in psoriatic arthritis: a systematic literature review. Arthritis Care Res 2012;64:397-406.

13. Orbai A-M, de Wit M, Mease P, Shea JA, Gossec L, Leung YY, et al. International patient and physician consensus on a psoriatic arthritis core outcome set for clinical trials. Ann Rheum Dis 2017;76:673-80.

14. Reygaerts T, Mitrovic S, Fautrel B, Gossec L. Effect of biologics on fatigue in psoriatic arthritis: a systematic literature review with meta-analysis. Joint Bone Spine 2017;85:405-10.

15. Tyring S, Gottlieb A, Papp K, Gordon K, Leonardi C, Wang A, et al. Etanercept and clinical outcomes, fatigue, and depression in psoriasis: double-blind placebo-controlled randomised phase III trial. Lancet 2006;367:29-35.

16. Ertenli I, Ozer S, Kiraz S, Apras S, Akdogan A, Karadag O, et al. Infliximab, a TNF-alpha antagonist treatment in patients with ankylosing spondylitis: the impact on depression, anxiety and quality of life level. Rheumatol Int 2012;32:323-30.

17. Orbai AM, Gladman DD, Goto H, Birt J, Lin CY, Kvien TK. Ixekizumab improves fatigue in a subset of patients with psoriatic arthritis [abstract]. Ann Rheum Dis 2018;77:1577.

18. Strand V, de Vlam K, Covarrubias-Cobos JA, Mease PJ, Gladman $\mathrm{DD}$, Graham D, et al. Tofacitinib or adalimumab versus placebo: patient-reported outcomes from OPAL Broaden - a phase III study of active psoriatic arthritis in patients with an inadequate response to conventional synthetic disease-modifying antirheumatic drugs. RMD Open 2019;5:e000806.

19. Strand V, de Vlam K, Covarrubias-Cobos JA, Mease PJ, Gladman DD, Chen L, et al. Effect of tofacitinib on patient-reported outcomes in patients with active psoriatic arthritis and an inadequate response to tumour necrosis factor inhibitors in the phase III, randomised controlled trial: OPAL Beyond. RMD Open 2019;5:e000808.

20. Hetland ML. DANBIO-powerful research database and electronic patient record. Rheumatology 2010;50:69-77.

21. Wolfe F. Fatigue assessments in rheumatoid arthritis: comparative performance of visual analog scales and longer fatigue questionnaires in 7760 patients. J Rheumatol 2004;31:1896-902.

22. Price DD, McGrath PA, Rafii A, Buckingham B. The validation of visual analogue scales as ratio scale measures for chronic and experimental pain. Pain 1983;17:45-56

23. Freynhagen R, Baron R, Gockel U, Tölle TR. Pain detect: a new screening questionnaire to identify neuropathic components in patients with back pain. Curr Med Res Opin 2006;22:1911-20.

24. Rifbjerg-Madsen S, Wæhrens EE, Danneskiold-Samsøe B, Amris K. Psychometric properties of the Pain Detect Questionnaire in rheumatoid arthritis, psoriatic arthritis and spondyloarthritis: Rasch analysis and test-retest reliability. Health Qual Life Outcomes 2017;15:110

25. Hochman J, Davis A, Elkayam J, Gagliese L, Hawker G. Neuropathic pain symptoms on the modified pain detect correlate with signs of central sensitization in knee osteoarthritis. Osteoarthritis Cartilage 2013;21:1236-42.

26. Atzeni F, Boccassini L, Di Franco M, Alciati A, Marsico A, Cazzola $\mathrm{M}$, et al. Chronic widespread pain in spondyloarthritis. Reumatismo 2014;66:28-32 